LA W RENCE LIVERMORE N A TIO NAL LABORATORY
Assessing the Economic Value and Optimal Structure of Large-scale Energy Storage

A. Lamont

November 8, 2012 
This document was prepared as an account of work sponsored by an agency of the United States government. Neither the United States government nor Lawrence Livermore National Security, LLC, nor any of their employees makes any warranty, expressed or implied, or assumes any legal liability or responsibility for the accuracy, completeness, or usefulness of any information, apparatus, product, or process disclosed, or represents that its use would not infringe privately owned rights. Reference herein to any specific commercial product, process, or service by trade name, trademark, manufacturer, or otherwise does not necessarily constitute or imply its endorsement, recommendation, or favoring by the United States government or Lawrence Livermore National Security, LLC. The views and opinions of authors expressed herein do not necessarily state or reflect those of the United States government or Lawrence Livermore National Security, LLC, and shall not be used for advertising or product endorsement purposes.

This work performed under the auspices of the U.S. Department of Energy by Lawrence Livermore National Laboratory under Contract DE-AC52-07NA27344. 


\title{
Assessing the economic value and optimal structure of large-scale electricity storage
}

\author{
Alan D. Lamont
}

\begin{abstract}
Large-scale electricity storage can enable arbitrage between periods when electricity is abundant, and cheap, and periods when it is scarce. In principle, this could enable greater use of baseload generation, and encourage investments in intermittent capacity such as wind and solar. If storage is to penetrate the system, the marginal value of storage capacity must be high enough to enable investments in storage. To have a significant impact on investments in intermittent technologies, it must be large enough to affect the prices on the system. This research develops a theoretical framework to evaluate the marginal values of the components of a storage system, and to characterize the impact of storage on the price patterns in the system. The theoretical approach is applied to an example system to illustrate the changes in marginal values as storage penetrates the system, and the impact on system prices.
\end{abstract}

\section{Index Terms--}

Power systems, Energy storage, Power generation planning

\section{NOMENCLATURE}

\section{Subscripts and superscripts:}

$a=$ designates generators

$c=$ designates the charging device for storage

$d=$ designates the discharging device for storage

$r=$ designates the storage reservoir

$h=$ designates an hour of the year

$j=$ designates a charge/discharge cycle

Objective function:

$C^{t o t}=$ total annual capital plus operating cost of the system, $\$ / y r$

\section{Decision variables:}

$k_{a}=$ the capacity of generator $a$, which is the peak output available from generator $a, \mathrm{~kW}$.

$k_{c}=$ capacity of the storage charge device, $\mathrm{kW}$

$k_{d}=$ capacity of the storage discharge device, $\mathrm{kW}$

$k_{r} \quad=$ capacity of the storage reservoir, $\mathrm{kWh}$

$g_{a, h}=$ output, or dispatch, of generator $a$ in the hour $h, \mathrm{~kW}$

$g_{d, h}=$ output, or dispatch, of storage discharge device in the hour $h, \mathrm{~kW}$

$g_{c, h}=$ output, or dispatch, of the storage charge in the hour $h$. This is the rate of energy input to the storage reservoir, $\mathrm{kW}$

$f_{j} \quad=$ the hour that the storage reservoir is fully charged on

Alan D. Lamont is an engineer at Lawrence Livermore National Laboratory, Livermore, California, 94550 (email: lamont1@1lnl.gov) cycle $j$

$e_{j}=$ the hour that cycle $j$ begins, with storage level at 0

Model parameters:

$C_{a}^{c a p}=$ annual capital cost of one unit of capacity for generator a or for the charge and discharge devices, $\$ / \mathrm{kW}$-yr

$C_{c}^{c a p}=$ annual capital cost of one unit of capacity for the storage charge device, $\$ / \mathrm{kW}-\mathrm{yr}$

$C_{d}^{c a p}=$ annual capital cost of one unit of capacity for the storage discharge device, $\$ / \mathrm{kW}-\mathrm{yr}$

$C_{r}^{c a p}=$ annual capital cost for one unit of reservoir capacity, $\$ / \mathrm{kW}$-yr

$C_{a}^{\mathrm{var}}=$ variable operating cost of generator $a$, including the charge and discharge devices, $\$ / \mathrm{kWh}$

$C_{c}^{\mathrm{var}}=$ variable operating cost of the storage charge device, $\$ / \mathrm{kWh}$

$C_{d}^{\mathrm{var}}=$ variable operating cost of the storage discharge device, $\$ / \mathrm{kWh}$

$D_{h}=$ demand in hour $h, \mathrm{~kW}$

$\eta_{d}=$ efficiency of the discharge device

$\eta_{c}=$ efficiency of the charge device

$F_{a, h}^{p r d}=$ the fraction of peak output (production factor) that generator $a$ can provide in period $h$. The production factor of intermittent generators varies from hour to hour. If $F_{a, h}^{p r d}=1.0$, the available output of the generator equals its peak capacity. If $F_{a, h}^{p r d}=0.5$, the available output is 0.5 of its peak capacity. For a dispatchable generator $F_{a, h}^{p r d}=1$ in all hours.

Variables:

$\lambda, \gamma, \alpha, \beta$

$=$ Lagrange multipliers, defined in the text.

Sets and references to elements of sets:

$H_{a}^{*}=$ the set of hours when generator $a$ is dispatched to its full available capacity.

$H_{c}^{*}=$ the set of hours when the charging device is dispatched to its full capacity

$H_{d}^{*}=$ the set of hours when the discharging device is dispatched to its full capacity

$H=$ all hours in a year.

$J=$ indices of all charging/discharging cycles.

$j(h)=$ the fill/empty cycle that contains hour $h$ 


\section{INTRODUCTION}

It is frequently suggested that large-scale energy storage will be an important part of a future, low carbon energy system. Proposed future energy systems based on large renewables either explicitly or implicitly assume that there will be storage available to make efficient use of the intermittent power generation through arbitrage between periods of high energy availability and periods when energy is scarce (Hoffman, et al [1], Jacobson and Delucchi [2], Turner [3]).

Renewable generation and storage can form a complementary system if they are economically complementary. That is, if the penetration of one technology tends to encourage investment in the other. As an example, wind often generates overnight when demand is low. Large wind capacity tends to drive down the prices discouraging further investment in wind. If large-scale storage is added to the system, it can charge during periods of low prices, raising the load on the system and increasing prices. The increased prices will encourage further investment in wind. However, the increased prices while charging will increase the cost of storage operation and limit the economic penetration of storage. To evaluate the economic role of storage we must address two questions:

- How is the penetration of storage determined by conditions on the system, and what is the most efficient configuration of capacities for generation, charging, discharging, and energy storage?

- How does large-scale storage affect the pattern of prices on the system and, thereby, affect the economic penetration of other technologies?

This paper develops an analytic optimization model to address these issues. The solution to the model's equations provide analytic expressions for the economic value of storage capacity investments, and provides insight into the patterns of price impacts that storage will have on the system.

The rest of the introduction describes the analytical approach for addressing these questions, and compares this approach to other work in the field. The details of the analytic model and its solution are discussed in Section III. Section IV provides a practical illustration of the application of the model to show the nature of the results that would be obtained. Section $\mathrm{V}$ discusses the price effects of storage and examines the impact that storage may have on renewable investments.

\section{A. Overview of the analytic approach:}

The approach first formulates an analytic model for the optimal configuration and operation of an electric generation system that includes storage. This is formulated as a Lagrangian optimization model ${ }^{1}$ to minimize the total capital and operating costs of the system. The equations are solved to derive analytic expressions for the conditions that determine the system price in each hour, the system dispatch, and the

\footnotetext{
${ }^{1}$ See Schweppe et al [4] for a similar Lagrangian formulation (without storage). The time horizon in the Schweppe formulation is the life of the investments whereas for this formulation the time horizon is a single year.
}

conditions for optimal investment in storage and generation capacity.

A key concept in capacity optimization is the "marginal value" of capacity. Heuristically, one can think of capacity as being added to a system in increments. Each incremental investment adds some amount of value to the system (for example, storage can decrease the cost of the system by using more low cost energy and displacing higher cost generation). The value of a small increment of capacity is the marginal value of capacity (strictly speaking, the term "marginal value" refers to infinitesimal increments of capacity). Typically the initial increments of capacity have a high marginal value, but the marginal value decreases as more capacity is added. As long as the marginal value is greater than the marginal cost it is optimal to add an increment of capacity. Once enough capacity has been added such that the marginal value is just equal to the marginal cost, the level of capacity is optimal. An essential result from optimization theory is that marginal value should equal marginal cost for all of the different types of capacity in the system (Schweppe [4], Stoft [5]).

The solutions to the Lagrangian model's equations provide analytic expressions for the marginal value of each of the components of the system (charge and discharge capacity, energy storage capacity, and generation capacity). These expressions demonstrate the changes in marginal value as storage penetrates the system. The process of finding an overall optimum is complicated by the fact that the changing level of capacity for one technology can change the marginal values of other technologies. The equations provide insight into these cross impacts. Finally, the expressions can be interpreted to provide insight into the pattern of prices that would result from energy storage.

The marginal value of capacity for any of the technologies depends on the way that it is actually used in the system. In particular, marginal value depends on the number of times the full capacity is used during the year and the value that additional capacity would contribute each time the full capacity is used. If the installed capacity is never fully used, then, obviously, adding more capacity does not contribute any value. Conversely, if the full capacity is used frequently, then it is likely that adding capacity could be valuable. In the case of storage, additional capacity would only be useful in those instances when the storage capacity is completely charged and the completely discharged. In those instances additional capacity would allow an operator to charge with more energy at a low price and discharge it at a higher price.

The marginal value of energy storage also depends on the price differential between charging and discharging each time the storage cycles. The value of storage tends to decrease as capacity is added because storage tends to raise system prices (system costs) when it charges, and lower system prices when it discharges. As more capacity is added, these price effects become stronger, narrowing the differential. Consequently the analysis of storage value needs to account for the price impacts of storage as capacity is increased.

In general, the charge/discharge power capacity of a storage system can be sized separately from the energy storage 
capacity. Since the charging and discharging capacity is a substantial part of the cost of a storage system ([6], [7]), the costs and values of the charging/discharging capacity have a substantial effect on the design of the system. The analysis finds separate expressions for the marginal values of these components.

The practical illustration portion of this paper applies this analysis to an example based on the California electricity grid. The example uses the equations from the theoretical analysis to compute the marginal values of charge/discharge capacity and storage capacity as their capacities are increased. These results can be used to determine the optimal capacities of storage capacity. Given the marginal costs of capacity we can find the level of capacity such that marginal cost equals marginal value, which will be the optimal set of capacities.

\section{B. Relation to previous work}

A number of studies have assessed the economic viability of electricity storage in different markets (EPRI [8], Eyer et al [9], Fertig and Apt [10], Hessami and Bowly [11]), Iannucci et al [12], Korpaas, et al [13], Lamont [14], NYSERDA [15]). These studies considered small-scale storage-systems that are small enough that they do not affect operation or prices of the electric system as a whole. The studies evaluated the net revenues that would accrue to the owner of a storage facility of a given capacity and compared them to the cost of installing and operating the storage facility. Analysis of small-scale systems gives a good understanding of the possibilities for initial penetration of storage. These essentially tell us the cost point at which an investor might be willing to invest in the first storage facility in a given market. But, they do not tell us how much capacity would be optimal.

As noted above, an understanding of the marginal value of capacity is essential for finding the optimal design of a storage system. Both [14] and [16] make empirical estimates of the marginal value of energy storage capacity by computing the total value of the storage system for a sequence of storage capacities. Computing the incremental change in total value per incremental change in storage capacity provides an estimate of the marginal value of storage capacity as a function of the total capacity. However, this empirical approach does not provide a basic understanding of the factors that determine the marginal value of storage capacity.

The discussion above addressed the fact that storage tends to raise prices when it charges and decrease prices when it discharges. This is an important effect both for the economics of the storage itself, and for the effect that storage will have on the economics of other technologies. The price impact of storage has been included in some studies (e.g. NYSERDA [15]). Sioshansi et al [16] estimates the price impact of largescale storage in order to evaluate the welfare implications of changing prices in the PJM system. However, these do not assess the impact of the changing prices on the economic viability of storage.

Tuohy and O'Mally [17] examine the possible changes to the entire system that storage would allow. By adjusting the generating capacity on the balance of the system, they illustrate scenarios in which the addition of storage reduces capital and operating costs of the balance of the system. These savings can be compared to the costs of the storage system to determine if storage is economically advantageous.

We note that storage might play a number of economically useful roles in a system aside from arbitrage. Among them are: regulation, congestion relief, and transmission deferral (Eyer et al, [9]). In these applications the prices will be determined by locational marginal prices, which are functions of generation costs, transmission losses, and transmission constraints. The same general form of analysis could be applied to these applications, however, the determination of prices would be more complex.

\section{ANALYTIC MODEL OF LARGE-SCALE STORAGE}

This analysis starts from a capacity optimization model of the system. Figure 1 illustrates the conceptual structure of the model. There is a set of generators plus a storage system. These are dispatched each hour to meet the demand. The storage is charged from the generators. The storage system consists of: 1) the storage reservoir (this might be a literal reservoir in the case of pumped hydro storage, a mass of chemical compound, a tank of hydrogen, or a spinning flywheel), 2) the charging device and 3) the discharging device. In the case of electro-chemical charging and discharging devices consist of wiring and possibly structures for heat dissipation. In the case of pumped hydro, charging and discharging is done through a pump-generator. For compressed air energy storage, it is a compressor (usually part of a combustion turbine).

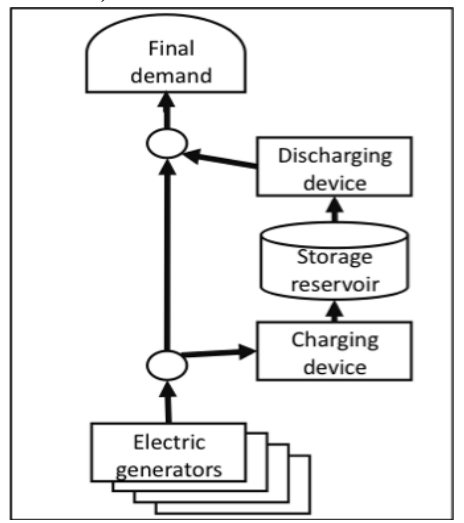

Figure 1: Schematic of energy storage in the generation system

Constraints in the optimization model require that: a) the demand be met each hour, b) the dispatch of the generators, the charging device, and the discharging device is always less than or equal to their capacities, and c) that the storage reservoir neither overcharges nor discharges below empty.

The mathematical analysis here treats the charging and discharging devices as separate devices. However, it is easy to deal with the case where they are the same device.

As is discussed above, the marginal value of a technology depends on the number of times that its capacity is fully used. In terms of the mathematical model, this is the number of 
times that the constraint on the dispatch of the technology is binding. When analyzing the marginal value of storage, we account for the cycles in the year that the storage is completely charged and then completely discharged, that is, the number of times that its capacity is fully used. Figure 2 illustrates the charging and discharging of storage and shows the variables used to characterize the cycling of the storage. We identify the cycles by the subscript $j$. The discussions below refer to the "charging intervals" and the "discharging intervals". As illustrated in the figure, these are the intervals when the storage goes from empty to fully charged, and then from fully charged to empty, respectively. The storage starts off empty at hour $e_{j}$. It then charges until completely full by hour $f_{j}$. To complete the cycle, it completely discharges by hour $e_{j+1}$.

During a charging or discharging interval, the energy stored might increase and decrease several times, but the cycle is only complete once the storage has completely charged and then completely discharged. The storage operator will earn additional revenue by partially cycling the storage (otherwise there would be no point in the partial cycles). However, these partial cycles do not contribute to the marginal value of the storage since the operator would earn the same revenue whether the capacity of the storage was increased or not.

The hours at which the storage completely charges or completely discharges are decision variables in the problem. There are corresponding constraints that the storage must completely charge and completely discharge by the hours specified.

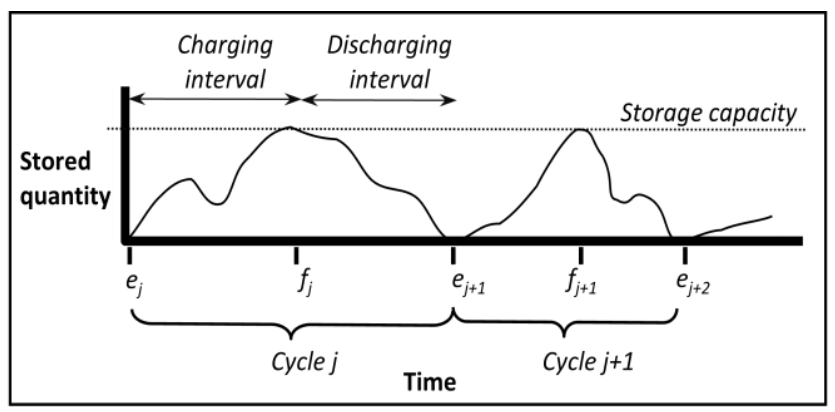

Figure 2: Illustration of the nomenclature for charging and discharging cycles

\section{A. Analytic model}

The model finds the capacities of the generators, capacities of storage system, the dispatch of the generators, and the times to charge and discharge the storage so as to minimize the total annual capital and operating cost of the system. The minimization is subject to the constraints that the total output each hour must equal the demand that hour, the dispatch of each generator must be less than or equal to its available capacity, and the storage must complete each cycle by fully charging and discharging.

The generators are denoted in the equations by the subscript $a$. The generators (and the storage discharge) are dispatched to meet the final demand in each hour, $D_{h}$. Some of the generation technologies are intermittent so that in some hours their available capacity is only a fraction, $F_{a, h}^{p r d}$, of their nameplate (or peak) capacity. For a dispatchable generator, $F_{a, h}^{p r d}$ is 1.0 for each hour (see Lamont [18] for details of the formulation to accommodate intermittent generation in an optimization model). This analysis ignores forced outages.

The analytic model is formulated as follows:

Objective: Minimize the total annual capital costs and annual operating cost

$$
\begin{aligned}
& C^{\text {tot }}=C_{a}^{c a p} \quad k_{a} \div+C_{c}^{c a p} \quad k_{c}+C_{d}^{c a p} \quad k_{d}+C_{r}^{\text {cap }} \quad k_{r} \\
& a \\
& +{ }_{h a}\left(C_{a}^{\mathrm{var}} g_{a, h}\right)+{ }_{h}\left(C_{c}^{\mathrm{var}} g_{c, h}\right)+{ }_{h}\left(C_{d}^{\mathrm{var}} g_{d, h}\right)
\end{aligned}
$$

Subject to the following constraints:

- Do not exceed capacity of any generator

$$
g_{a, h}=F_{a, h}^{p r d} \quad k_{a} \quad a, h \quad H_{a}^{*}
$$

This constraint would usually be entered into an optimization model as $g_{a, h} F_{a, h}^{p r d} k_{a}$. However, the marginal value of capacity is zero when the constraint is not binding, and those hours drop out of the analysis. For the purpose of exposition, it is simpler to omit those hours from the constraint equations and only include the hours when the constraint is binding and generation is equal to available capacity. We denote the set of hours that the constraint is binding for generator $a$ as $H_{a}^{*}$.

- Do not exceed capacity of the charging device

$$
g_{c, h}=k_{c} \quad h \quad H_{c}^{*}
$$

- Do not exceed capacity of the discharging device

$$
g_{d, h}=k_{d} \quad h \quad H_{d}^{*}
$$

- Meet the demand in all hours, including the charging demand

$$
g_{a, h}+g_{d, h}=D_{h}+\frac{g_{c, h}}{\mathrm{c}} \quad h \quad H
$$

- Storage reservoir must charge to capacity each cycle:

$\mathrm{f}_{\mathrm{j}}$

$g_{h=\mathrm{e}_{\mathrm{j}}} g_{c, h} \frac{g_{d, h}}{d} \div=k_{r} \quad j \quad J$

- Storage reservoir must discharge to zero each cycle $\mathrm{e}_{\mathrm{j}+1}$

$$
\underset{h=\mathrm{f}_{\mathrm{j}}}{\frac{g_{d, h}}{d}} g_{c, h} \div=k_{r} \quad j \quad J
$$

The complete Lagrangian is: 


$$
\begin{aligned}
& L=C_{a}^{c a p} k_{a}+C_{c}^{c a p} k_{c}+C_{d}^{c a p} k_{d}+C_{r}^{c a p} k_{r} \\
& \text { a } \\
& +{ }_{h a}\left(C_{a}^{\mathrm{var}} g_{a, h}\right)+{ }_{h}\left(C_{c}^{\mathrm{var}} g_{c, h}\right)+{ }_{h}\left(C_{d}^{\mathrm{var}} g_{d, h}\right) \\
& +{ }_{h} D_{h}+\frac{g_{c, h}}{\mathrm{c}} g_{d, h} \quad g_{a, h} \underset{\mathrm{a}}{\stackrel{\dot{\leftrightarrows}}{\dot{亠}}} \text { meet demand } \\
& +\quad a, h g_{a, h} \quad F_{a, h}^{p r d} \quad k_{a} \div \quad \text { do not exceed generator capacities } \\
& a h H_{a}^{*} \\
& +\quad c, h\left(\begin{array}{ll}
g_{c, h} & k_{c}
\end{array}\right) \\
& \text { do not exceed charging capacity } \\
& h H_{c}^{*} \\
& +\underset{h H_{d}^{*}}{d, h\left(g_{d, h} \quad k_{d}\right)} \\
& \text { do not exceed discharging capacity }
\end{aligned}
$$

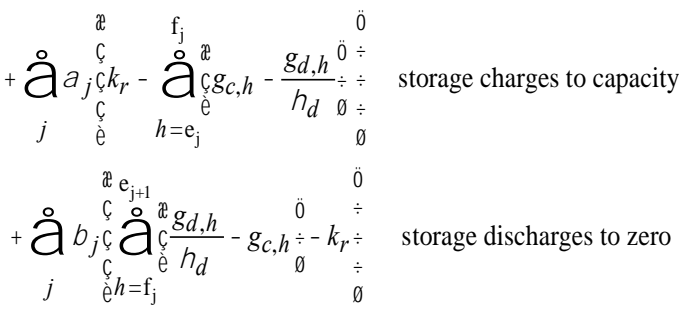

\section{B. The derivatives of the Lagrangian and their interpretations}

To solve the Lagrangian, we take the derivatives with respect to each of the variables and set them to zero. The resulting equations have interpretations for the optimal operation of the system and for the computation of the marginal values of the components of the system.

In these interpretations, the Lagrange multipliers play a prominent role. To clarify the following discussion, the interpretation of the multipliers is discussed below:

- $\lambda_{h}$ is the Lagrange multiplier for the constraint that the sum of the output from the generators plus the discharge of the storage must equal the demand in hour $h . \lambda_{h}$ is the system marginal cost (SMC) in hour $h$. It equals the marginal generating cost of the most expensive generator that has been dispatched. It is the reduction in cost that would result from reducing the demand by one unit. In a competitive market the system marginal cost is the system price (see Stoft [5], CAISO [19], or Kirschenand and Strbac[20]).

- $\gamma_{a, h}, \gamma_{c, h}, \gamma_{d, h}$, are the multipliers for the constraints that the dispatch of the generators, the charge device, or the discharge device cannot exceed the capacities. These are the reductions in cost that would result from increasing the capacity of those units in hour $h$.

- $\alpha_{j}$ is the multiplier for the constraint that the storage must completely charge in cycle $j$. It is the marginal system cost of adding one more unit of energy to storage in cycle $j$. If another increment of capacity were added to the storage reservoir, it would cost $\alpha_{j}$ to fill that unit in cycle $j$.

- $\beta_{j}$ is the multiplier for the constraint that the storage cannot discharge below zero during cycle $j$. It is the marginal value of releasing an additional unit of energy from the storage reservoir. If another unit of capacity were added to the storage reservoir, the system cost would be reduced by $\beta_{j}$, and the storage operator would earn $\beta_{j}$, when the energy from that unit was released in cycle $j$. Note that this refers to a unit of energy taken from the reservoir and passed to the discharge device. Because of discharge efficiency losses, less than one unit of energy will actually be discharged into the system.

The next sections present the derivatives of the Lagrangian with respect to each of the variables in the analysis and interpret them in terms of the operation of the system and the marginal values of capacity of the components.

1) Optimal operation of the generators: Derivatives with respect to the generation, $g_{a, h}$ :

This provides the conditions for optimal operation of the generators. Note that the storage charging and discharging devices are not included here since their conditions are somewhat different. Differentiating the Lagrangian with respect to $g_{a, h}$ for each hour and setting the results to zero yields:

$$
\begin{array}{ll}
\lambda_{h}=C_{a}^{\mathrm{var}} & h \notin H_{a}^{*} \\
\gamma_{a, h}=\lambda_{h}-C_{a}^{\mathrm{var}} & h \in H_{a}^{*}
\end{array}
$$

The condition in Equation (9a) applies for those hours when generator $a$ is not dispatched to its full available output. The generator should be dispatched such that its marginal operating cost is equal to $\lambda_{h}$, the marginal system cost (SMC) in that hour (as noted above, SMC is the system price in an efficient market). The variable costs in this formulation are constant so a generator is dispatched if its variable cost is less than or equal to $\lambda_{h}$, and is not dispatched otherwise.

Equation (9b) shows the derivatives for those hours that the generator is dispatched to its full capacity. The system marginal cost will be greater than, or equal to, the generator's marginal operating cost. The difference between the generator's marginal operating costs and the system marginal cost is $\gamma_{a, h}$. This is the value that an additional unit of capacity would provide in that hour, provided it generates at full capacity. As long as generator $a$ is not the marginal generator, an additional unit of capacity would allow us to back down the marginal generator, saving a cost of $\lambda_{h}$ (the generation cost of the marginal generator), and costing $C_{a}^{\mathrm{var}}$. The net savings is $a, h={ }_{h} \quad C_{a}^{\mathrm{var}}$.

2) Optimal dispatch of charging: Derivative with respect to the rate of charge, $g_{c, h}$

This derivative provides the conditions for the optimal dispatch of the charging device in each hour. These conditions indicate the level of dispatch of the charging device and the contribution to the marginal value of the charging capacity in hour $h$. At hour $h$, the system could be in one of four different system conditions depending on whether or not the charging device is dispatched to capacity in hour $h$ (so that the constraint is binding) and whether or not the system is in the charging or discharging interval of a cycle during the hour $h$. 
Depending on the condition, different terms of Equation (8) apply, giving a different derivative for each of the four conditions. Differentiating the Lagrangian with respect to $g_{c, h}$ and setting to zero provides the following set of conditions to met in hour $h$ :

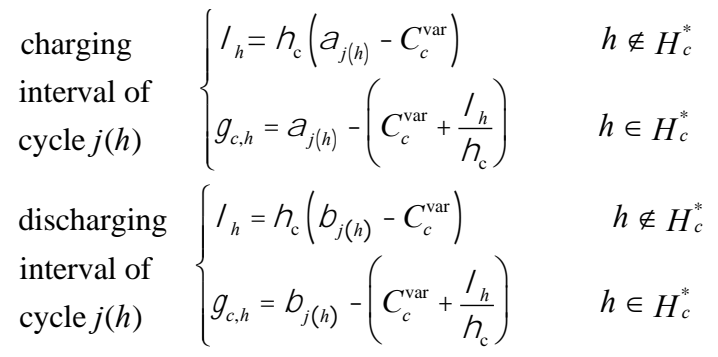

In these equations $\alpha$ is the marginal cost of adding a unit of energy to the storage (after accounting for efficiency and any operating costs). That is, it is the cost of the most expensive energy that is added during cycle $j$. If $\alpha$ is the marginal cost, then the operator will begin to charge storage any time the

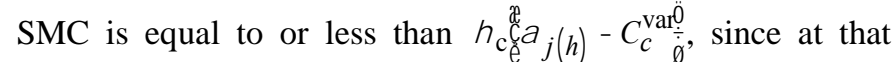
SMC, the cost of adding a unit of energy to the storage is $\alpha$.

Figure 3 illustrates the behavior of storage charging and SMCs, (or prices), during a charging interval. At the start of the interval the end-use demands on the system are relatively high so the SMC is greater than ${ }^{c} j(h) C_{c}^{\mathrm{var}} \div$ and the storage does not charge. As we move to later hours, the enduse demands decrease allowing the SMC to decrease. If there were no storage, the SMC would follow the fine line at the bottom of the graph.

At hour a the SMC declines to $h={ }_{c} \quad j(h) \quad C_{c}^{\mathrm{var} \div}$ and the storage begins to charge. Initially the charging device is not fully dispatched so equation 10a applies. As the storage charges, it adds load on the system, raising the SMC. Between hours $a$ and $b$, it places enough additional load on the system to maintain the SMC at $\mathrm{c} j(h) C_{c}^{\mathrm{var}} \div$ as required by equation (10a).

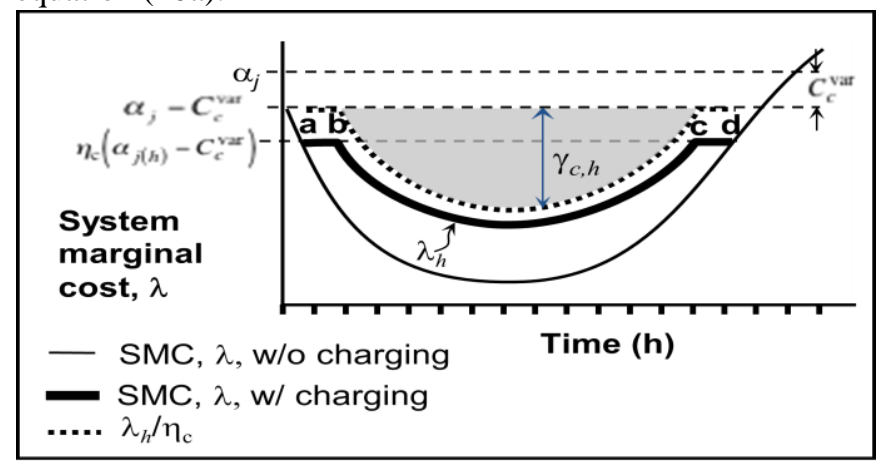

Figure 3: Illustration of changes in system marginal cost during a charging interval

The hours between $a$ and $b$ and between $c$ and $d$ are the marginal hours of charging during this cycle-any additional units of energy stored will be added in these hours since the charging device is dispatched to capacity in other hours. As is discussed below, the marginal costs of charging and discharging have a central role in determining the marginal values of charging capacity and reservoir capacity.

At hour $b$ the end-use demands on the system drop to the point that the charging device is fully dispatched and can no longer maintain the $\mathrm{SMC}$ at $\quad$ c $\quad j(h) \quad C_{c}^{\mathrm{var}} \stackrel{\div}{\circ}$ At this point the SMC begins to decline further, following the heavy line. From hour b until hour $\mathrm{c}$ the charging device is fully dispatched and Equation (10b) applies. During this interval, additional charging capacity would be valuable to the system since it would allow the storage to take in more energy at a lower price. Equation (10b) computes the contribution to the marginal value of charging capacity in each hour. Just as in the case of other conversion technologies, $\gamma_{c, h}$ is the Lagrange multiplier on the constraint that the capacity of the charging device cannot be exceeded. Here $\gamma_{c, h}$ is the difference between, $j(h)$ the marginal cost of adding to storage in that cycle, and $C_{c}^{\mathrm{var}}+h / c$, the cost of adding a unit of energy in that hour. With another increment of charging capacity, the storage could add a unit of energy at this hour (when the price is low) and avoid adding a unit of energy at the marginal cost. This difference is the value that additional charging capacity would provide in that hour. The marginal value of a unit of charging capacity is the sum of the $\gamma \mathrm{s}$ over the year. For a single charging cycle, as illustrated in Figure 3, the contribution to the marginal value of charging capacity is the area of the grey semicircle bounded by $j(h) C_{c}^{\mathrm{val}}$ at the top and the heavy dashed line labeled $h / \mathrm{c}$.

The discussion up to now refers to charging during the charging interval. It is also possible for the storage to charge during the discharge interval. Equations (10c) and (10d) address the case. Typically the system price is high during the discharge interval (hence the storage discharges). However, If the SMC drops below ${ }_{h}={ }_{c}\left(\begin{array}{ll}j(h) & \left.C_{c}^{\mathrm{var}}\right)\end{array}\right)$ it will be

worthwhile to charge. The cost of adding a unit of energy to the reservoir is ${ }_{h} /{ }_{c}+C_{c}^{\text {var }}$ which is just equal to the value of releasing a unit of energy from the reservoir, $j(h)$ for cycle $j$

(as noted above, due to efficiency losses, less than one unit will actually discharged to the system). By charging, storage will cause the system price to rise. If the storage has enough charging capacity, it should charge at a rate that raises the system price to ${ }_{h}={ }_{c}\left(\begin{array}{ll}j(h) & C_{c}^{\mathrm{var}}\end{array}\right)$ as indicated by Equation

(10c). Equation (10d) addresses the case where the charging capacity is not large enough to raise the system price to

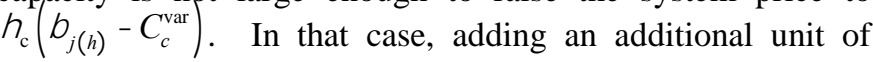

charging capacity would allow the operator to charge at a cost that is less than the earnings that he will receive from releasing the unit of energy. The difference is $\gamma_{c, h}$, which is the marginal value of additional charging capacity in that hour. 


\section{3) Optimal dispatch of discharging: Derivatives with}

respect to the rate of discharge each hour, $g_{d, h}$

This derivative gives us the conditions for the optimal dispatch of the discharging device for each hour. Differentiating with respect to $g_{d, h}$ and setting the derivatives to zero gives the following equations governing the discharge of storage and the contributions to the marginal value of the discharge capacity. Similar to the charging device there are four different system conditions that give us four different equations:

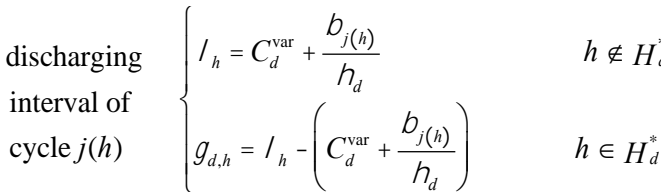

$$
\begin{aligned}
& \begin{array}{ll}
\text { charging } \\
\text { interval of } \\
\text { cycle } j(h)
\end{array} \begin{cases}{ }_{h}=C_{d}^{\mathrm{var}}+\frac{j(h)}{d} & h \notin H_{d}^{*} \\
{ }_{d, h}={ }_{h}\left(C_{d}^{\mathrm{var}}+\frac{j(h)}{{ }_{d}}\right) & h \in H_{d}^{*}\end{cases}
\end{aligned}
$$

The behavior of the system marginal cost during discharge is illustrated in Figure 4. This is analogous to the behavior during charging. During the discharge part of the cycle the storage begins to discharge once the SMC increases to $C_{d}^{\mathrm{var}}+{ }_{j(h)} / d$. At this SMC, $\beta_{j}$ is the marginal earnings per unit released from the storage reservoir (less than one unit will be discharged to the system due to the efficiency losses of the discharging device).

Analogous to the charging cycle, between hours $a$ and $b$ and between $\mathrm{c}$ and $\mathrm{d}$ the storage discharges at a rate to maintain the SMC at $C_{d}^{\mathrm{var}}+j(h) / d$. Between hours $\mathrm{b}$ and $\mathrm{c}$ the load on the system rises to the point that the discharge device is dispatched to capacity and can no longer maintain that SMC. After time $b$ the SMC rises following the heavy line.

In each hour between $\mathrm{b}$ and $\mathrm{c}, \gamma_{d, h}$, is the marginal value of discharging capacity. It is the difference between the SMC, $\lambda_{h}$, and $C_{d}^{\mathrm{var}}+j(h) / d$ in equation (11b). The logic is as follows: An additional unit of discharge capacity would allow the operator to discharge another unit at hour $h$ and earn ${ }_{h} C_{d}^{\text {var }}$. Discharging that unit of energy would remove $1 / \eta_{\mathrm{d}}$ units of energy from the reservoir, after accounting for the efficiency losses of discharging. The operator would forego releasing that energy at the marginal hour, so he would forego earnings of $j(h) / d$. The net revenues in hour $h$ from another unit of discharging capacity would be ${ }_{d, h}={ }_{h} C_{d}^{\mathrm{var}}{ }_{j(h)} / d$. In Figure 4, the area of the grey semicircle bounded by the SMC (with charging, the heavy line) on the top and the line $C_{d}^{\mathrm{var}}+{ }_{j}(h) /{ }_{d}$ on the bottom is the total contribution to the marginal value of discharge capacity for this cycle.

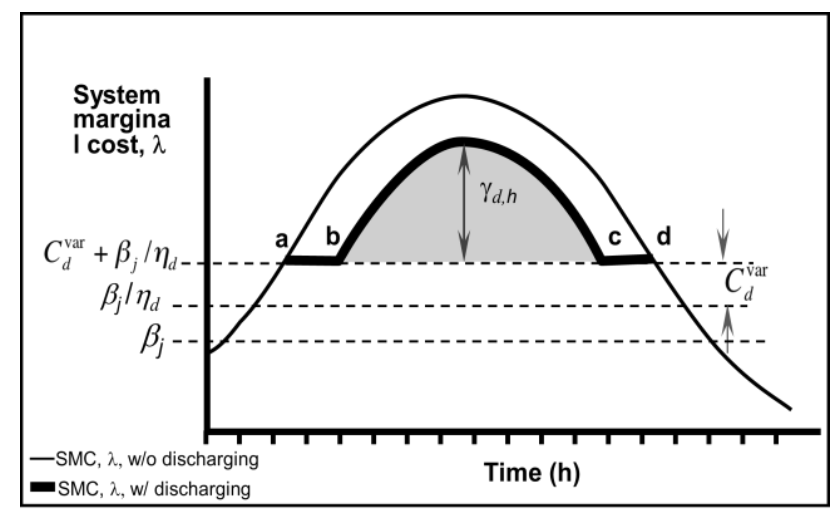

Figure 4 : Illustration of system marginal cost during discharge interval

4) Capital cost recovery of charge and discharge devices, and the generators: Derivatives with respect to the capacities, $k_{c}, k_{d}$, and $k_{a}$

The derivatives with respect to the capacities give the conditions for the capital cost recovery of the charge and discharge devices, and the generators. Setting these derivatives to 0 yields the following equations:

$$
\begin{aligned}
& C_{c}^{c a p}={ }_{h H_{c}^{*}}^{c, h} \\
& C_{d}^{c a p}={ }_{h H_{d}^{*}}^{d, h} \\
& C_{a}^{c a p}={ }_{h H_{a}^{*}}\left(\begin{array}{ll}
a, h & F_{a, h}^{p r d}
\end{array}\right)
\end{aligned}
$$

To optimize system, capacity is added to each type of technology up to the point that the marginal value of capacity is equal to the marginal cost of capacity. The total marginal value of capacity is the sum of the hourly marginal capacity values, $\gamma \mathrm{s}$, over the year.

To account for intermittent generators the available capacity in each hour is multiplied by $F_{a, h}^{p r d}$ (the production factor for the generator in hour $h$ ) so the actual value provided by an increment of capacity is $a, h \quad F_{a, h}^{p r d}$ in hour h. Recall that the production factor for a dispatchable generator is 1 .

5) Optimal energy storage capacity: Derivative with respect to the capacity of the storage reservoir, $k_{r}$

This derivative gives us the optimal conditions for the capital cost recovery of the storage reservoir

Setting the derivative to 0 yields:

$$
{ }_{j=1}^{J}(j \quad j)=C_{r}^{c a p}
$$

From equation (15) we see that the marginal value of the reservoir is a function of the number of times that the reservoir fully cycles over the year $(J)$ and the differentials between the marginal costs of charging $(\alpha)$ and the marginal value of discharging $(\beta)$ when it cycles. Note that the number of cycles in the year is determined by the optimization. 
6) Case when the same device charges and discharges

In this mathematical formulation charging and discharging are represented as separate devices. In many real storage technologies charging and discharging use the same physical device. When that is the case, the marginal value of capacity is simply the sum of the hourly marginal capacity values $(\gamma s)$ attributed to the device in either mode.

\section{Interactions between capacities and marginal values of storage system components}

The previous equations show the mechanisms that lead to the changes in marginal values of the components as the capacities of the components are changed. There is a selfeffect, the marginal value of a component decreases as its own capacity increases, and there is a cross-effect, the marginal value of one component increases when the capacity of another component is increased.

As an example, consider the effects of increasing the storage reservoir capacity. When the capacity of the storage reservoir is increased, it must charge over more hours in order to completely fill (given that charging capacity does not change). This implies that $\alpha$, which governs the system price at which it begins to charge, must be increased for each cycle. From Equation (15) it is seen that this will decrease the marginal value of the reservoir capacity. In addition, increasing the capacity of the reservoir may reduce the number of times that it can completely cycle, further reducing the marginal value.

However, when $\alpha$ is increased due to an increase in the reservoir capacity, the marginal value of charging capacity is increased in each hour that the reservoir charges and the reservoir will charge for more hours. This can be most easily seen in Figure 3. The height and width of the shaded area increase, which corresponds to an increase in the marginal value of the charging capacity.

\section{PRACTICAL ILLUSTRATION OF THE APPLICATION AND THE MARGINAL VALUES OF THE STORAGE SYSTEM COMPONENTS}

The following example uses the equations derived above to illustrate the marginal value and penetration of large-scale storage in an example based on data for California. It is not intended to be a model of the California system, but this gives a view of the results that could be obtained from the theoretical analysis derived above. The example computes the marginal values of charging/discharging capacity and reservoir capacity for different combinations of capacities. Given the costs and efficiencies of these capacities for a given storage technology, we can determine the optimal capacities and penetration in the system.

\section{A. Structure of the modeled system and the underlying data}

This example is based on the hourly prices from a time dependent value study of the California system (PG\&E, [21]) and loads (CAISO [22]) for 2001. The price pattern over the year is shown in Figure 5. The peak load in this example has been scaled to $60 \mathrm{GW}$ and the total generation over the year is $332,000 \mathrm{GWh}$. It assumes that the storage is $90 \%$ efficient on charging and discharging (81\% efficient round trip). It also assumes charging and discharging use the same device.

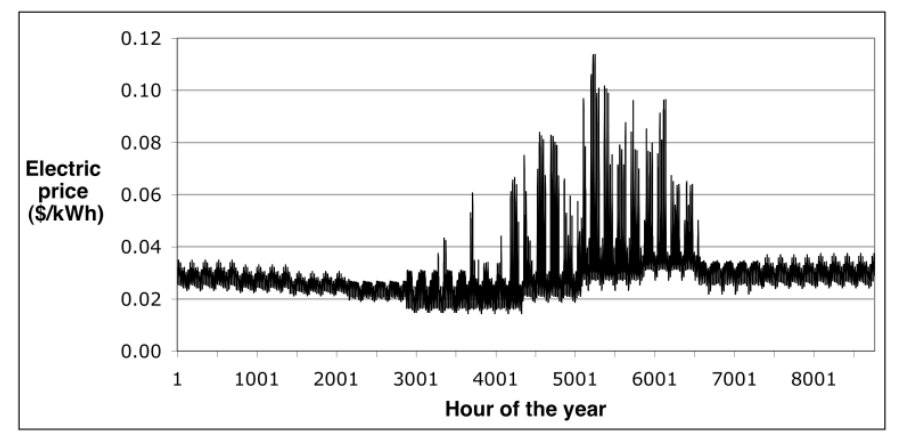

Figure 5: Patterns of prices over the year for this example

\section{B. Modeling steps}

The analysis first develops a relationship between load and price on the system. It then applies the equations above to determine the charging or discharging each hour of the year. This gives us the values of $\alpha$ and $\beta$ for each cycle and the marginal values of capacity. The final step plots the contours of marginal values for storage reservoir capacity and charge/discharge capacity as a function of the reservoir and charge/discharge capacity.

The relationship between price and load is modeled using a bi-linear fit for each day. One linear fit is calibrated to the low load hours of the day and the other is calibrated for the highest hours of load each day. During most months the two linear fits are very similar. During the summer months there is a sharp break with rapidly rising prices once the load exceeds a threshold. The threshold is different for each day, so a different fit is needed for each day. Figure 6 illustrates the bi-linear fit for a summer day.

A series of cases was run assuming different values for storage energy capacity and charge/discharge power capacity in each case. For each case, the model was executed for each day over the year. The Solver function in Excel ${ }^{\mathrm{TM}}$ was used to determine the values of $\alpha$ and $\beta$. For each case the $\alpha \mathrm{s}, \beta \mathrm{s}$ and $\gamma$ s were summed up over the year to give the marginal values of capacities for that case.

To implement the model $\alpha_{j}, \beta_{j}$, and $J$ must be determined. If it was economically advantageous, the storage was completely cycled each day. In the algorithm values for $\alpha_{j}$ and $\beta_{j}$ needed to fully charge and discharge for each day were first determined. The values of $\alpha_{j}$ and $\beta_{j}$ implicitly determine the hours at which the storage reservoir will completely charge and completely discharge.

As long as the spread of SMC was great enough, $\alpha_{j}$ was less than $\beta_{j}$ and it was economically worthwhile to cycle the storage fully on that day. In some cases the SMC spread was so small that $\alpha_{j}$ would have to be greater than $\beta_{j}$ in order to fully cycle the storage. This implies that if the storage were completely charged and discharged that day, the marginal cost of charging would be greater than the marginal revenue of 
discharging. Clearly this would not be efficient. In those cases the storage was only cycled up to the point that $\alpha_{j}$ was equal to $\beta_{j}$. During these partial cycles the storage system can earn net revenues. But these revenues do not contribute to the marginal value of storage capacity since the storage system could have earned the same revenue with less capacity. The number of times that the storage could completely cycle over the year determined the value of $J$.

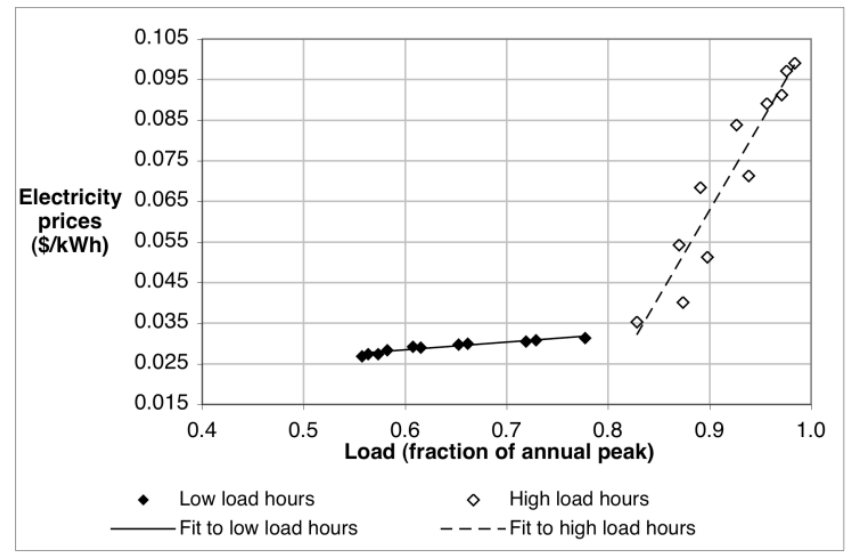

Figure 6: Example of bilinear fit on day 220

\section{Results from the example analysis}

The primary result from the analysis are shown in Figure 7 which shows two super-imposed sets of contours, one set for the marginal values of reservoir capacity and the other set for the marginal values of charge/discharge capacity. These are plotted as functions of the reservoir energy capacity and charge/discharge power capacity.

From Figure 7 we can determine the optimal configuration of a storage system. In an optimized system, the marginal cost of a technology should be just equal to its marginal value. For a given type of storage technology, the marginal costs of reservoir capacity and charge/discharge capacities are known as a function of the total capacity deployed (for most technologies the marginal cost is constant). For a given marginal cost, we know that the optimal solution will fall on the contour of the corresponding marginal value. We can determine the contours that correspond to marginal costs of reservoir and charge/discharge capacity. At the point where the two contours intersect the marginal values of reservoir capacity and of charge/discharge capacity will be just equal to their marginal costs. This gives the optimal capacities for that storage technology. To illustrate, assume (optimistically) that there is a storage technology with an annual cost $2.0 \$ / \mathrm{kW}$-yr for the charge/discharge capacity and a reservoir cost of cost of $1.5 \$ / \mathrm{kWh}$-yr. The optimal storage system for this example would have a charge/discharge capacity of $1.25 \mathrm{GW}$ and a reservoir capacity of about 6.3 $\mathrm{GWh}$. If the annual cost of the reservoir were to decline to 1.0 $\$ / \mathrm{kWh}$-yr, the optimal capacities would be $2.2 \mathrm{GW}$ of charge/discharge capacity and $12.5 \mathrm{GWh}$ of reservoir capacity.

As would be expected, both types of capacity show decreasing returns to scale. The figure also illustrate the cross effects between capacities and marginal values of the storage components. Increasing the capacity of one component improves the marginal value of the other component. At very small capacities this effect is very strong. As a result, the two sets of contours are fairly parallel for small values of reservoir and charge/discharge capacity.

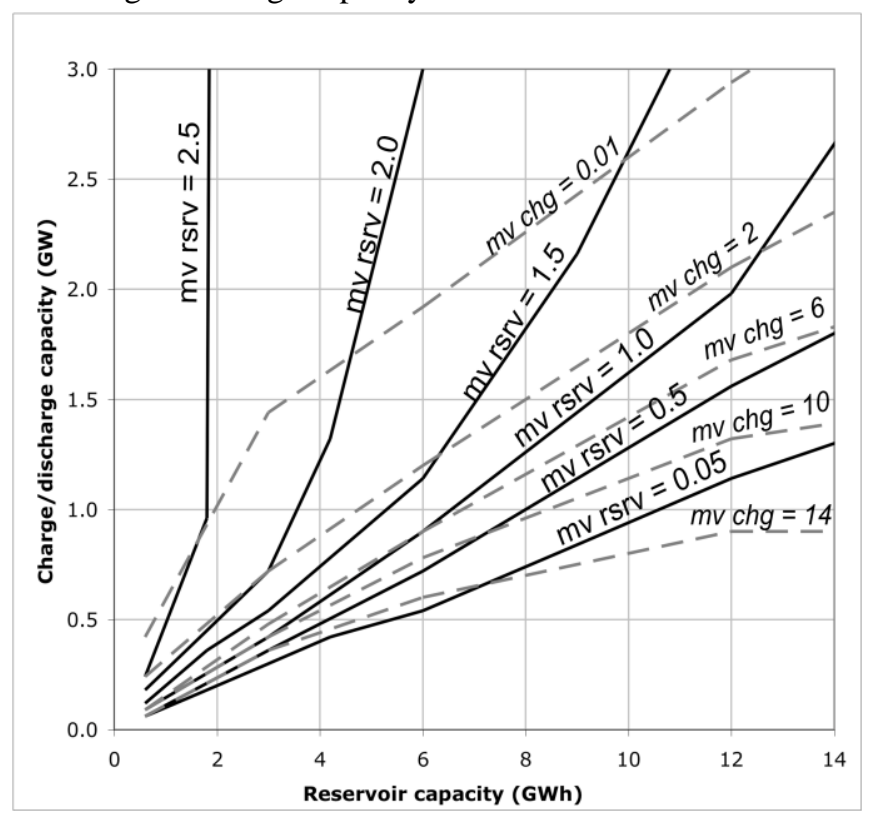

Figure 7: Contours of the marginal values of storage reservoir capacity ( $\mathrm{mv} \mathrm{rsvr}, \$ / k W h-y r)$ and charge/discharge capacity (mv chg, $\$ / k W-y r)$

\section{IMPACTS OF STORAGE ON RENEWABLE INVESTMENTS}

The illustration computes the prices that result from the operation of storage. The price duration curve in Figure 8 provides insight regarding the economic interaction between storage and renewable generation. This figure is constructed assuming large capacities for charge/discharge and storage (10 $\mathrm{GW}$ of charge/discharge and $30 \mathrm{GW} / \mathrm{h}$ of storage capacity) in order to make the results clearer.

As noted in the introduction, storage could increase the penetration of, say, wind if it tended to raise prices in off-peak hours when wind is generating but prices are low. The results of this example indicate that storage would have a relatively small effect on off-peak prices since the off-peak prices are not particularly responsive to demands, as illustrated in Figure 6. This could often be the case since off-peak naturally implies that there are substantial generation resources available.

Figure 8 also indicates that storage substantially reduces the on-peak prices. Again, figure 6 indicates that on-peak prices are quite sensitive to loads. In a summer peaking system such as California's, solar generators earn a substantial portion of their revenues during the peak hours of the day. Reducing the prices in peak hours would tend to discourage investment in solar technologies. 


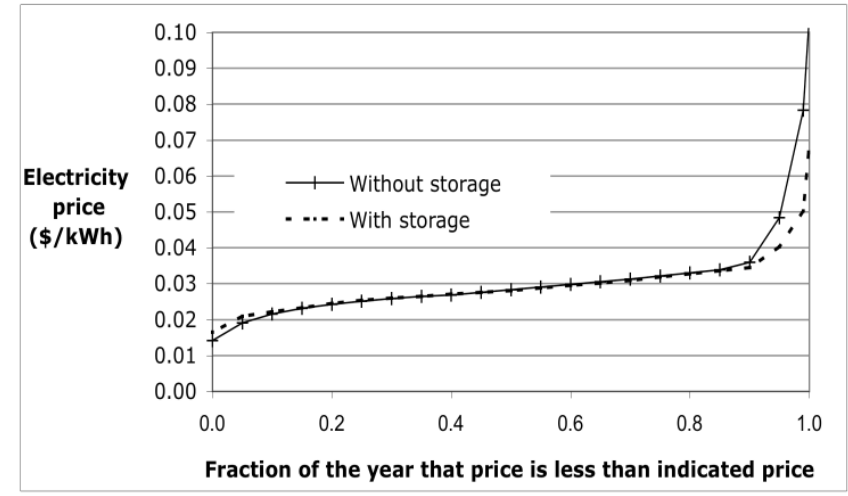

Figure 8: price duration curves with and without storage (assumes $30 \mathrm{GWh}$ of reservoir storage and $10 \mathrm{GW}$ of charge/discharge capacity)

\section{CONCLUSIONS}

If storage is to encourage investments in intermittent renewable resources, it will be deployed at scales large enough to affect the prices on the system. The change in prices affects both the ultimate penetration of storage itself and the economic penetration of other technologies. This work provides the theoretical framework to determine the optimal operation of storage, evaluate its effect on system marginal prices, and assess the marginal value of the storage components.

Applying this theory to an example system illustrates several points: 1) the use of these equations to optimize the capacities of the storage system, 2) the interaction between marginal values of charge/discharge capacity and the storage reservoir capacity, and 3) the impact that storage might have on the penetration of renewable generation.

\section{ACKNOWLEDGMENT}

The author would like to thank Tom Edmunds and Yiming Yao for their review of the manuscript and for their helpful comments, suggestions, and questions.

\section{REFERENCES}

[1] I. Hoffman, A. Byrne, D. Kammen, "Redefining What's Possible for Clean Energy by 2020: Job Growth, Energy Security", Climate Change Solutions, June 2009

[2] M. Z. Jacobson, M. A. Delucchi, "A path to sustainable energy by 2030", Scientific American, November 2009

[3] J. A. Turner, , "A Realizable Renewable Energy Future”, Science, Vol. 285. no. 5428, July 1999: pp. $687-689$

[4] F. C. Schweppe, , M. C. Caramanis, R. D. Tabors, and R. E. Brown, Spot Pricing of Electricity, Kluwer Academic Publishers, 1987

[5] S. Stoft, Power systems economics, Wiley Interscience, 2002

[6] D. Boutacoff, "Energy storage: Emerging strategies for energy storage", IEEE Power Engineering Review, December 1989,

[7] T. B. Johansson, H. Kelly, A. K. N. Reddy, R. H. Williams, eds., L. Burnham, exec. ed., Renewable energy, sources for fuels and electricity, Island Press, 1993

[8] EPRI, Market driven distributed energy storage system requirements for load management applications. EPRI, Palo Alto, CA, 1014668, 2007

[9] J. M. Eyer, J. J. Iannucci, G. P. Corey, Energy storage benefits and market analysis handbook, Sandia National Laboratories, SAND20046177,2004
[10] E. J. Fertig, J. Apt, "Economics of compressed air energy storage to integrate wind power: A case study in ERCOT, Energy Policy, 39, 2011, pp. $2330-2342$

[11] M-A Hessami, D.R. Bowly, "Economic feasibility and optimization of an energy storage system for Portland Wind Farm (Victoria, Australia), Applied Energy, 88, 2011, pp 2755-2763

[12] J. Iannucci, J. Eyer, B. Erdman, Innovative applications of energy storage in a restructured electricity marketplace Phase III final report, Sandia National Laboratories, SAND2003-2546, 2003

[13] M. Korpaas, A. T. Holena, R. Hildrumb, "Operation and sizing of energy storage for wind power plants in a market system", Electrical Power and Energy Systems, Vol. 25, 2003, pp 599-606

[14] A. D. Lamont, Improving the value of wind energy generation through back-up generation and energy storage, December, California Energy Commission report CEC-500-2005-183, 2005

[15] NYSERDA, Guide to estimating benefits and market potential for electricity storage in New York (with emphasis on New York City), New York State Energy Research And Development Authority, Final Report 07-06, March 2007

[16] R. Sioshansi, P. Denholm, T. Jenkin, J. Weiss, "Estimating the value of electricity storage in PJM: Arbitrage and some welfare effects", Energy Economics, Vol. 31, 2009, pp 269-277

[17] A. Tuohy, M. O'Mally, "Pumped storage in systems with very high wind penetration", Energy Policy, 39, 2011, pp.1965 - 1974

[18] A. D. Lamont, "Assessing the long-term system value of intermittent electric generation technologies", Energy Economics, v. 30, Issue 3, May 2008

[19] CAISO (California Independent System Operator), Technical Bulletin 2009-06-05, Market Optimization Details, June 16, 2009 revised November 19, 2009

[20] D. Kirschenand G. Strbac, Fundamentals of power systems economics, John Wiley and Sons, 2004

[21] PG\&E, Time Dependent Valuation of Energy for Developing Building Efficiency Standards, Time Dependent Valuation (TDV) Formulation 'Cookbook', prepared for PG\&E by Heschong Mahone Group and Energy and Environmental Economic, March 2001

[22] CAISO, (California Independent System Operator) OASIS data base, http://oasis.caiso.com/ , accessed May 12, 2004

\section{BIOGRAPHY}

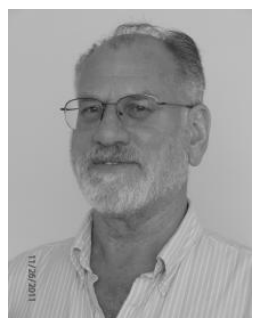

Alan D. Lamont graduated from Stanford University in 1970 with Bachelors and Masters degrees in Civil Engineering. After a term with the Peace Corps in Venezuela designing smal dams, he worked for Woodward-Clyde Consultants in earthquake analysis for nuclear power plants and pipeline construction in Alaska. He returned to Stanford, and completed a PhD in Engineering Economic Systems in 1983. From 1983 to 1987 he was part of the Decision Analysis Group at Woodward-Clyde. Since 1987 he has been with Lawrence Livermore National Laboratory working in analysis of security systems, decision analysis, risk analysis, and the economics of energy systems. 